\title{
A Review on Role of Active Contour Model in Image Segmentation Applications
}

\author{
R.S. Jaiswal ${ }^{1}$, M.V. Sarode ${ }^{2}$ \\ Department of CSE, COET, Akola, $\mathrm{MH}^{1}$ \\ Department of CSE, Government Polytechnic, Yavatmal, $\mathrm{MH}^{2}$
}

\begin{abstract}
In recent years, Active contours have been widely studied and applied in image analysis. Active contours combine underlying information with high-level prior knowledge to achieve automatic segmentation for complex objects. Their applications include edge detection, segmentation of objects, shape modelling and object boundary tracking. This paper presents the development process of active contour models and describes the classical parametric active contour models, geometric active contour models, and new hybrid active contour models based on curve evolution and energy minimization techniques. It also discusses challenges and applications of active contour models in image segmentation.
\end{abstract}

Keywords: parametric model, geometric model, active contour.

\section{INTRODUCTION}

Image segmentation is a fundamental issue in biomedical imaging area. Segmenting structures from medical images and the reconstruction of a compact analytic representation of these structures is difficult. Medical images are often corrupted by noise and sampling artifacts, which can cause considerable difficulties when applying classical segmentation techniques such as edge detection and thresholding. As a result, these techniques either fail completely or require some kind of post processing step to remove invalid object boundaries in the segmentation results. To address these difficulties, active contour method have been extensively studied and widely used in medical image segmentation, with promising results. The segmentation operation can carried out manually or automatically. A manual segmentation requires a skilled operator trained to use a digital tool to mark the contours of the desired structures. An obvious disadvantage is that an exhaustive process, where the results are hardly repeatable. Automatic techniques usually apply evolving interfaces dynamically adaptable to the desired features contained in the image. The difference between the two techniques is whether or not the user is involved the process.

In this paper, we are mainly concerned about the application active contour model in image segmentation. In next section we gives an overview theoretical background of the active contour model.

\section{ACTIVE CONTOUR MODEL}

The active contour model / Snakes are a special form of deformable models, and are characterized by their property of dynamic deformation to an image from an original given shape. The active contour models are visually represented as closed contours (like an irregular balloon or bubble).

Deformable models are curves or surfaces defined within an image domain that can move under the influence of internal forces, which are defined within the curve or surface itself, and external forces, which are computed from the image data. The internal forces are designed to keep the model smooth during deformation. The external forces are defined to move the model toward an object boundary or other desired features within an image.

The active contour model is an energy minimizing spline; with it's energy dependent upon its shape and location within an image. Typically, local energy minima correspond to desired image properties. The energy function to be minimized is a weighted combination of internal and external energy forces. Snakes lend themselves to object delineation, particularly where guided by higher level understanding processes (whether interaction with human or guidance from an automatic system). At the very least, they require a starting shape and location, preferably near to that of the desired object. Snakes can be open (like a length of string) or closed (more like balloons).

Enode $=\operatorname{Eint}(\mathrm{v}(\mathrm{s}))+\operatorname{Eimage}(\mathrm{v}(\mathrm{s}))+$

Econstraint(v(s)) 
where $\mathrm{v}(\mathrm{s})$ represents a node on the curve, Eint the internal energy of the contour, Eimage the image forces , Econstraint the external constraints.

Summing the energy at every node gives the energy value for the snake as a whole.

The internal energy at any given node is combination of forces controlling elasticity and stiffness, and tends to produce smooth contours. These forces are calculated using the position of the node under consideration and its neighbours together with minimum and maximum values for such measures over the entirety of the active contour model.

The image energy of the snake at any given node is typically derived from some image processing technique, that is suitable for any chosen image domain, such as a first order edge gradient operator like the Sobel.

$$
E_{\text {int }}=f\left(\gamma^{\prime}(s)\right)+g\left(\gamma^{\prime \prime}(s)\right)
$$

The functions $f$ and $g$ are just going to be the Euclidean norm of the function. Then we need to specify that we want the energy for the whole curve $\mathrm{C}$ so not only for one spatial location s so we are going to sum this energy along the curve. Which will lead to two different cases.If our contour is closed:

$$
E_{\text {int }}=\oint_{\mathcal{C}}\left\|\gamma^{\prime}(s)\right\|^{2}+\left\|\gamma^{\prime \prime}(s)\right\|^{2} d s
$$

Or if we just consider a non closed contour going from A to B:

$$
E_{\text {int }}=\int_{A}^{B}\left\|\gamma^{\prime}(s)\right\|^{2}+\left\|\gamma^{\prime \prime}(s)\right\|^{2} d s
$$

Where $(\alpha, \beta) \varepsilon \mathrm{R}^{2}$ in a general definition but there are certain restrictions to their possible values.

There are two components to the internal energy measure at any node; both take into consideration the neighbouring nodes. The effect of these positive measures (elasticity and stiffness) is controlled by the elasticity and stiffness coefficients. As alpha is decreased (from a nominal value of 1.0), the strength of the elasticity of the contour at a node is decreased, giving nodes greater freedom in movement and so possibly producing more irregular contours. As beta is decreased (from a nominal value of 1.0), the stiffness of the contour at a node is decreased, allowing sharper corners to develop. It has been found through experimentation that any change in the elasticity co-efficient should typically be accompanied by a similar change in the stiffness coefficient.

The external forces affecting the active contour model are typically based on higher order constraints relating to more global strategies, such as the relation to other objects in the image or coercive forces forcing the snake towards or away from particular areas local to the snake. The provision of a means for specifying these external energy factors allows a degree of flexibility in the use of the active contour model for finding accurate contours in noisy or busy image areas.

$$
E_{\text {ext }}=P(\gamma(s))
$$

Where P stands for a potential attraction field onto the edge of an object. So the underlying idea here is to use an attraction field and a potential energy on that field that we will minimize to reach an edge of an object. So we have to consider this energy not only locally but for the whole contour $\mathrm{C}$ and to plug in the previous equation the properties we mentioned above.

If the contour is closed we have:

$$
E_{e x t}=\oint_{\mathcal{C}}\|\nabla I\|^{2}(\gamma(s)) d s
$$

Or if we just consider a non closed contour going from A to B:

$$
E_{e x t}=\int_{\Delta}^{B}\|\nabla I\|^{2}(\gamma(s)) d s
$$

Where $\mathrm{I}$ is representing the input image and $\nabla$ is the spatial gradient function defined by 


$$
\nabla I=\left(\frac{\partial I}{\partial x}, \frac{\partial I}{\partial y}\right)
$$

Due to the fact that we want to minimize this energy, we are going to take the opposed value and introduce our Gaussian smoothing to enforce convergence to a local minimum:

$$
E_{\text {ext }}=-\int_{A}^{B}\left\|\nabla\left(G_{n} * I\right)\right\|^{2}(\gamma(s)) d s
$$

Where Gn is a Gaussian weighted kernel of dimension $\mathrm{n}$.

Using a weighting parameter on the external energy will allow us to increase the "visibility" of the gradient field by the snakes. So we can write it :

$$
E_{e x t}=-\delta \int_{A}^{B}\left\|\nabla\left(G_{n} * I\right)\right\|^{2}(\gamma(s)) d s
$$

Where $\delta$ is a real weighting value which for obvious reason would be positive.

$$
\begin{aligned}
& E_{\text {snake }}=E_{\text {int }}+E_{\text {ext }} \\
& =\int_{A}^{B} \alpha\left\|\gamma^{\prime}(s)\right\|^{2}+\beta\left\|\gamma^{\prime \prime}(s)\right\|^{2} d s \\
& -\delta \int_{A}^{B}\left\|\nabla\left(G_{n} * I\right)\right\|^{2}(\gamma(s)) d s
\end{aligned}
$$

There are basically two types of deformable models : parametric deformable models and geometric deformable models. Parametric deformable models represent curves and surfaces explicitly in their parametric forms during deformation. This representation allows direct interaction with the model and can lead to a compact representation for fast real-time implementation. Adaptation of the model topology, however, such as splitting or merging parts during the deformation, can be difficult using parametric models. Geometric deformable models, on the other hand, can handle topological changes naturally. These models, based on the theory of curve evolution and the level set method, represent curves and surfaces implicitly as a level set of a higher-dimensional scalar function. Their parameterizations are computed only after complete deformation, thereby allowing topological adaptivity to be easily accommodated. Despite this fundamental difference, the underlying principles of both methods are very similar.

\section{PARAMETRIC DEFORMABLE MODELS}

In this section, we first describe two different types of formulations for parametric deformable models: an energy minimizing formulation and a dynamic force formulation. Although these two formulations lead to similar results, the first formulation has the advantage that its solution satisfies a minimum principle whereas the second formulation has the flexibility of allowing the use of more general types of external forces.

\subsection{Energy Minimizing Formulation}

The basic premise of the energy minimizing formulation of deformable contours is to find a parameterized curve that minimizes the weighted sum of internal energy and potential energy. The internal energy specifies the tension or the smoothness of the contour. The potential energy is defined over the image domain and typically possesses local minima at the image intensity edges occurring at object boundaries. Minimizing the total energy yields internal forces and potential forces. Internal forces hold the curve together (elasticity forces) and keep it from bending too much (bending forces). External forces attract the curve toward the desired object boundaries. To find the object boundary, parametric curves are initialized within the image domain, and are forced to move toward the potential energy minima under the influence of both these forces. 


\subsection{Dynamic force formulation}

It is used in those cases in which it is more comfortable to form deformable model straight from dynamic problem with help of force formulation. These formulations facilitate the use of common external forces, even those which are not potential, e.g. forces which can not be described as a negative gradient of potential energy function.

\section{GEOMETRIC MODELS}

It defines a front evolving according to a given curvature dependent speed function $\mathrm{F}$. This function can also depend on the image gradient. When the speed function is monotonic. We can define a geometric models based on the Level Set evolution curve.

\subsection{Level Set}

[Xin-Jiang et al.,2012] Level set is a numerical solution for processing topological changes of contours. Many of the PDEs used in image processing are based on moving curves and surfaces with curvature-based velocities. In this area, the level set method was very influential and useful. The basic idea is to represent the curves or surfaces as the zero level set of a higher dimensional hyper-surface. This technique not only provides more accurate numerical implementations but also handle topological change very easily.

Basically, it means that the closed curves in a two-dimensional surface are regarded as a continuous surface of a threedimensional space. The definition of a smoothing function $\phi(x, y, t)$ stands for the surface while the set of definitions $\phi(x, y, t)=0$, whose zero level set corresponds to curve. With the curve as the boundary, the whole surface can be divided into an internal region and an external region of the curve. Define a Signed Distance Function (SDF) on the surface:

$$
\phi(x, y, t=0)=d
$$

Where, the value of $d$ is the shortest distance between the point of $x$ on the surface and the curve. In the whole evolutional process of the curve, its points will fit into the following formula:

$$
\phi(x, y, t)=0
$$

The common movement formula of Level Set is:

$$
\phi_{t}+F|\nabla \phi|=0
$$

F is the speed function, which is a function related to evolving surface characteristics (e.g. curvature, normal direction, etc.) and image characteristics (e.g. gray, gradient). When applied into image segmentation, the design of $\mathrm{F}$ depends on the information of image and the ideal value is zero on the edge of the target (i.e. the bigger value of the gray gradient). Level set method, due to its stability and irrelevancy with topology, displays a great advantage in solve the problems of corner point producing, curve breaking and combing, etc. Therefore, it is used in a wide range. However, there are several disadvantages to this approach. Since the edge-stopping function depends on the image gradient, only objects with edges defined by gradient can be segmented. Another disadvantage is that in practice, the edge-stopping function is never exactly zero at the edges, and so the curve may eventually pass through object boundaries.

\section{APPLICATION of ACTIVE CONTOUR}

In this section we show how the previous active contour models, both parametric and geometric, can be applied to image segmentation.

[Kouta Fujimura et al., 1993] This paper describes a method of motion tracking and analysis of a deformable object in time-varying images. The approach is based upon modeling the boundary of the object as a new active contour model defined by an energy functional in terms of both intra- and interframe constraints, and then tracking the object boundary in consecutive image frames by minimizing the energy functional using dynamic programming in multiple scales. In order to increase the efficiency of the method, dynamic programming is carried out by using variable neighbors for each model point; that is, we define coarse neighbors at a coarse scale, and finer ones at a finer scale. The proposed motion tracking method has been successfully applied to the behavioral analysis of a slug in the biological study of learning and memory formation in slugs, and also to the problem of tracking the boundary of the left ventricle of the heart from time-varying ultrasonic echocardiographic images. In the former application, motion analysis is further carried out by tracking characteristic points which are detected as local curvature extrema along the object contour. In the latter application, the temporal change of the apparent area of target object region is computed in order to quantify cardiac motion. 
[M. Airouche et al., 2009] In this paper they explore image segmentation using active contours model to detect oil spills. A partial differential equation based level set method, which represents the spill surface as an implicit propagation interface, is used. Starting from an initial estimation with priori information, the level set method creates a set of speed functions to detect the position of the propagation interface. Specifically, the image intensity gradient and the curvature are utilized together to determine the speed and direction of the propagation. This allows the front interface to propagate naturally with topological changes, significant protrusions and narrow regions, giving rise to stable and smooth boundaries that discriminate oil spills from the surrounding water.

[Chendke and Hingoliwala, 2015] They present a framework for active contour-based visual object tracking using the level sets. The main components of their framework consist of the contour-based tracking initialization, colour-based contour evolution, the adaptive shape-based contour evolution for the non-periodic motions, the dynamic shape-based contour evolution for the periodic motions and handling of the abrupt motions. For the contour-based tracking initialization, they use an optical flow-based algorithm for the automatically initializing contours at the first frame. In the color-based contour evolution, they use Markov random field theory to measure correlations between values of the neighboring pixels for the posterior probability estimation. In the adaptive shape-based contour evolution, we combined the global shape information and the local color information to hierarchically develop gradually the contour, and a flexible shape updating model is made. In the dynamic shape based contour evolution, a shape mode transition matrix is gain to characterize the temporal correlations of the object shapes. In the handling of abrupt motions, particle swarm optimization (PSO) is used to capture the global motion which is applied to the contour in the current frame to produce an initial contour in the next frame.

[Amine Ben Slama et al., 2015] They presented a new method to resolve the problem of the estimation of eye position in the analysis of videonystagmography (VNG) sequences, that studies eye vibration using active contour model. An algorithm for horizontal and vertical nystagmus tracking based on some parameters such as position, amplitude and duration, is presented. Indeed, the algorithm uses active contour method to segment the pupil. Thus, segmentation is obtained from Otsu's thresholding, to detect an ellipse region approximates the pupil form. Then, the snake-model uses the estimated ellipse as the initial contour. This proposed approach is approved on clinical samples of the videonystagmography (VNG) from patient who presented a congenital nystagmus.

[Abdelhafid Nachour et al.,2017] In this paper, they develop a new method -called Fast Adaptive Balloon (FAB)sufficient to segment complex shape with lower computational complexity. The proposed definition for balloon force achieves satisfactory segmentation performance compared with other ACMs using both synthetic and medical images in two dimension. The results demonstrate the accuracy and effectiveness in segmentation besides the convergence speed.

\section{CONCLUSION}

The main conclusion from this work is that there is no ideal segmentation method. Both parametric and geometric active contours are driven by forces extracted from the image itself, what makes them extremely dependent on the image quality, that is, lowly noised, fair definition of the structures' edges and absence of local minima. Even if one is able to overcome these problems, there are still further difficulties, like the initialization problem for example, which has a strong impact on the correct contour's convergence. This kind of problem may cause the procedure to be repeated until the result obtained is good enough for the user.

\section{REFERENCES}

1. Kouta Fujimura et al.,1993] Kouta Fujimura, Naokazu Yokoya,Kazuhiko Yamamoto, "Motion Tracking of Deformable Objects by Active Contour Models Using Multiscale Dynamic Programming", in Journal of Visual Communication and Image Representation, Volume 4, Issue 4, December 1993.

2. M. Airouche et al., 2009] M. Airouche, L. Bentabet and M. Zelmat, "Image Segmentation Using Active Contour Model and Level Set Method Applied to Detect Oil Spills", in Proceedings of the World Congress on Engineering volume 1,2009.

3. Xin-Jiang et al.,2012] Xin-Jiang, Renjie-Zhang, Shengdong-Nie "“ Image Segmentation Based on Level Set Method”, in International Conference on Medical Physics and Biomedical Engineering,2012.

4. Chendke and Hingoliwala,2015] Suvarna D. Chendke, H. A.Hingoliwala "Active Contour-Based Visual Tracking by Integrating Colors, Shapes, and Motions Using Level Sets", in International Journal on Recent and Innovation Trends in Computing and Communication, volume: 3 Issue: 1 ,January 2015.

5. Amine Ben Slama et al.,2015]Amine Ben Slama, Ahmed Nejmedine Machraoui, Mohamad Sayadi, "Pupil tracking using active contour model for videonystagmography applications", Available: https://www.researchgate.net/publication/282739233_Pupil_tracking_using_ active_contour_model_for_videonystagmography_applications .

6. Abdelhafid Nachour et al.,2017]Abdelhafid Nachour ,Latifa Ouzizi, Youssef Aoura ,“ A Fast Adaptive Balloon Active Contour For Image Segmentation ",in Biomedical Engineering :Applications, Basis and Communications , volume 29, Issue 01, February 2017. 